\title{
Power system of the Guanay II AUV
}

\author{
Ivan Masmitjà, Julián González, Gerard Masmitjà, Spartacus Gomáriz, Joaquín del-Río-Fernández \\ SARTI Reserch Group, Electronics Dept., Universitat Politècnica de Catalunya (UPC), Rambla Exposició 24, 08800, Vilanova i la Geltrú. \\ Barcelona. Spain +(34) 938967 200. www.cdsarti.org
}

\section{ABSTRACT}

Guanay II is an autonomous underwater vehicle (AUV) designed to perform measurements in a water column. In this paper the aspects of the vehicle's power system are presented with particular focus on the power elements and the state of charge of the batteries. The system performs both measurement and monitoring tasks and also controls the state of charge (SoC) of the batteries. It allows simultaneous charging of all batteries from outside the vehicle and has a wireless connection/disconnection mode. Guanay II uses a NiCd battery and for this reason the current integration as a SoC methodology has been selected. Moreover, it has been validated that it is possible to obtain instant consumption from the SoC circuit. Finally, laboratory and vehicle navigation tests have been performed to validate the correct operation of the systems and the reliability of the measured data.

\section{Section: RESEARCH PAPER}

Keywords: State of charge; batteries; AUV; Wireless connection; Ni-Cd

Citation: Ivan Masmitjà, Julián González, Gerard Masmitjà, Spartacus Gomáriz, Joaquín del-Río-Fernández, Power system of the Guanay II AUV, Acta IMEKO, vol. 4, no. 1, article 7, February 2015, identifier: IMEKO-ACTA-04 (2015)-01-07

Editor: Paolo Carbone, University of Perugia

Received December $12^{\text {th }}, 2013$; In final form November $8^{\text {th }}, 2014$; Published February 2015

Copyright: (C) 2014 IMEKO. This is an open-access article distributed under the terms of the Creative Commons Attribution 3.0 License, which permits unrestricted use, distribution, and reproduction in any medium, provided the original author and source are credited

Funding: This work was supported by the Spanish ministry of economy and competitiveness under the research project: "Sistemas inalambricos para la extension de observatories submarinos" (CTM2010-15459)

Corresponding author: Ivan Masmitjà, e-mail: ivan.masmitja@upc.edu

\section{INTRODUCTION}

The study of the sea and oceans has become increasingly important among fishers and biologists especially because marine species change their behavior depending on environmental variables such as temperature, salinity and ph, among others [1]. For these oceanographic observations different tools are used. Aerospace technologies can focus on the study of the oceans at a global level but are unable to carry out detailed observations in a specific sector or depth. Oceanographic ships, on the other hand, can solve this problem but the actual planning and mission deployment required to obtain a satisfactory special-time resolution data are very expensive. As a solution to these needs, these tools are migrating towards the creation of autonomous marine vehicles.

The autonomous underwater vehicle (AUV) Guanay II [2] [4] is a vehicle developed by the SARTI group of the Technical University of Catalonia (with the co-financing of IMEDEA-CSIC) with the objective to provide a platform for measuring oceanographic variables, such as temperature and salinity of the water column, with a high simultaneously spatial and temporal resolution. Figure 1 shows the vehicle. The knowledge of the charge status of the batteries in an autonomous vehicle is an important factor to ensure security of the vehicle and mission. This work presents a measurement system and energy

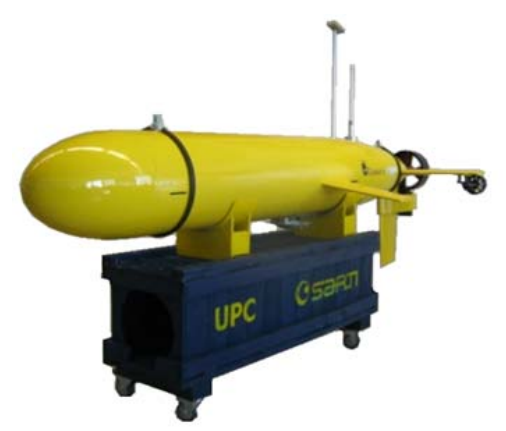

Figure 1. Autonomous Underwater Vehicle Guanay II AUV. 
management for the Guanay II vehicle. Initially different solutions for electrically measuring the batteries charge state are analyzed, and the methodology of current integration is chosen [5] due to the nature of the batteries used in the Guanay II. Subsequently, the construction of a prototype and the experimental tests performed both in laboratory and field missions in order to validate the correct operation of the device is presented. Finally, this device is complemented with a design of a battery charger system, accessible from outside the vehicle, which allows simultaneous charging of all battery packs, and also a wireless connection-disconnection of the battery.

\section{POWER SYSTEM OF GUANAY II AUV}

The Guanay II's power system can be divided into three parts: propulsion system; communication and control system; and battery system. Below, each of these elements are briefly described.

The propulsion systems consist of a series of propellers for longitudinal and directional movement and a motorpiston set to modify the buoyancy of the vehicle to perform dives. The principal characteristics of the main engine, for longitudinal movement, are: $24 \mathrm{~V}$ of direct current (dc) supply; 300W of output power; and can be controlled via RS232 communication. Two engines are located on the rear side of the vehicle to control its direction. The engines have $24 \mathrm{~V}$ of power supply, maximum power output of $110 \mathrm{~W}$ each and are controlled by an RS232 communication port. Finally, the pistonengine set comprises a motor of $24 \mathrm{Vdc}$ and a piston that can move up to 1.5 litres. This piston can either take in or eject seawater. With this action the buoyancy of the vehicle can change and therefore the dives can be controlled. These systems consume most of the Guanay II AUV power.

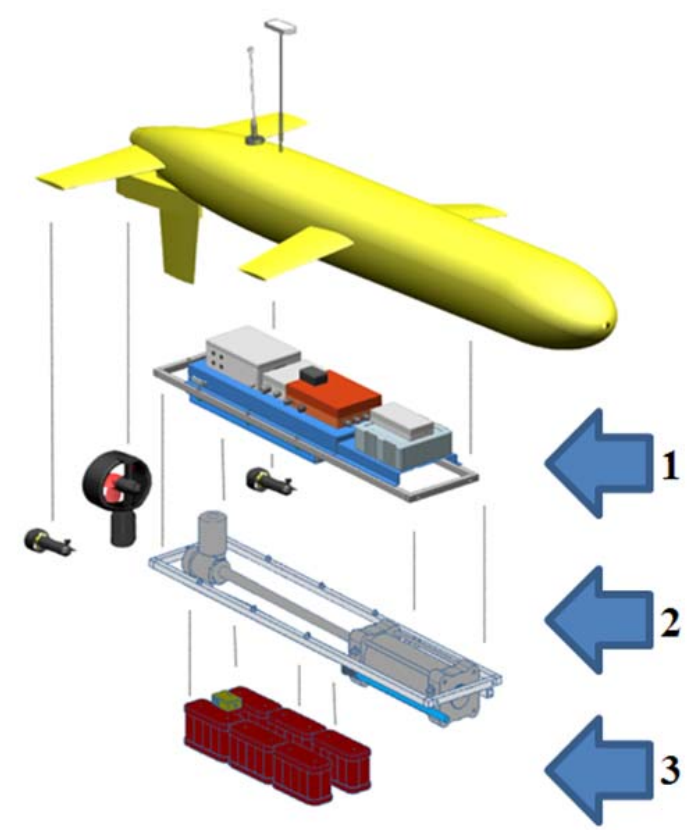

Figure 2. This figure shows the development of the vehicle in 3D with the three parts of the power system (1, Communication and control system. 2 , Propulsion system. 3, Battery system).
Table 1. Theoretical consumptions of the Guanay II.

\begin{tabular}{c|ccc}
\hline & \multicolumn{3}{|c}{ Maximum values } \\
\hline & $\mathrm{V}$ & $\mathrm{A}$ & $\mathrm{W}$ \\
\hline GPS & 5 & $240 \mathrm{~m}$ & 2 \\
PC104 & 5 & 0.9 & 9.9 \\
Compass & 5 & $<20 \mathrm{~m}$ & 0.1 \\
Radio-modem & 12 & 1.5 & 18 \\
Engine Driver & 5 & $31 \mathrm{~m}$ & 0.15 \\
Main Thruster & 24 & 16 & 300 \\
Lateral Thruster & 24 & 4.25 & 110 \\
Piston-Engine & 24 & 3 & 50 \\
\hline TOTAL & \multicolumn{3}{|c|}{} \\
\hline
\end{tabular}

The second part of Guanay II vehicle is the control and communication system. The main control system of the vehicle is an embedded computer located inside. This computer is responsible for controlling and managing the various elements of the vehicle (sensors, actuators, motors, communications, etc.) Also, by using a radio link and a WiFi network the vehicle can be remotely controlled from a base station.

Finally, an energy storage system has been mentioned. This vehicle has autonomy of around 4 hours thanks to the nickel-cadmium (Ni-Cd) battery pack of $24 \mathrm{~V}$ and $21 \mathrm{Ah}$. This pack consists of a subset of $12 \mathrm{Vdc}$ and $7 \mathrm{Ah}$ batteries arranged in two series and three in parallel configuration (2s3p). With these batteries and the average consumption of electronic components of the vehicle, the autonomy under normal conditions can be established.

Figure 2 shows the development of the vehicle in 3D where the three parts of the power system can be seen (1, Communication and control system. 2, Propulsion system. 3, Battery system).

Finally, in Table 1 the values of the theoretical consumption of various devices of the vehicle can be seen. These values are specified in the technical specifications of the devices.

As seen in table 1, the engines consume most (90\%) of the vehicle's energy. The other electronic systems consume the remaining $10 \%$. Given these data and the total capacity of the batteries, the autonomy of the vehicle can be known. If the engines run at full power, a maximum of less than 1.5 $\mathrm{h}$ has been obtained. However, various factors have to be taken into account. First, the side engines correct the direction of the vehicle, but are never on for long time. With regard to the main engine, a compromise between speed and autonomy of the vehicle is necessary. Finally, the engine piston set is only activated to make the dives. In experimental results, it has been observed that the vehicle has a range of about 4 hours under normal conditions.

\section{MEASUREMENT SYSTEM FOR BATTERY STATE OF CHARGE}

The state of charge (SoC) is mathematically defined as in the following equation (1).

$\operatorname{SoC}(t)=\left(A h_{\text {nom }}-\int_{0}^{t} I(t) d t\right) / A h_{\text {nom }}$ 
where $I(t)$ is the current extracted from the battery (which is assumed positive while discharging the battery), and $A h_{\text {nom }}$ is the nominal battery capacity. Equation (1) assumes that the current integration starts at $\operatorname{SoC}(\mathrm{t})=100 \%$ at $t=$ 0 .

Several methods of estimating the SoC of a battery have been used. Some are specific to particular cell chemistries. This work is subject to the use of $\mathrm{Ni}-\mathrm{Cd}$ batteries used in the Guanay II.

\subsection{Methods for SoC estimation}

For such batteries there are different methods to measure the charge, such as the measurement of voltage, impedance and current integration.

a. Voltage based SoC estimation uses the voltage of the battery cell as the basis for calculating the remaining capacity [6]. Results can vary widely depending on discharge rate and temperature and compensation for these factors must be provided to achieve reasonable accuracy. Figure 3 shows the relationship between the voltage and the capacity at different discharge rates.

b. Internal impedance measurements can also be used to determine the SoC. However these are not widely used because of difficulties in measuring the impedance while the cells are active as well as difficulties in interpreting the data and very complex calculations are required [7] and [8].

c. Current based SoC estimation, known as coulomb counting [5] [11]. In coulometric measurements however, the amount of capacity taken out or put into a battery is measured in terms of ampere-hour or in \%. The coulomb counting approach basically implements equation (1) to evaluate the SoC. It uses a more general definition, defined as can be seen in (2).

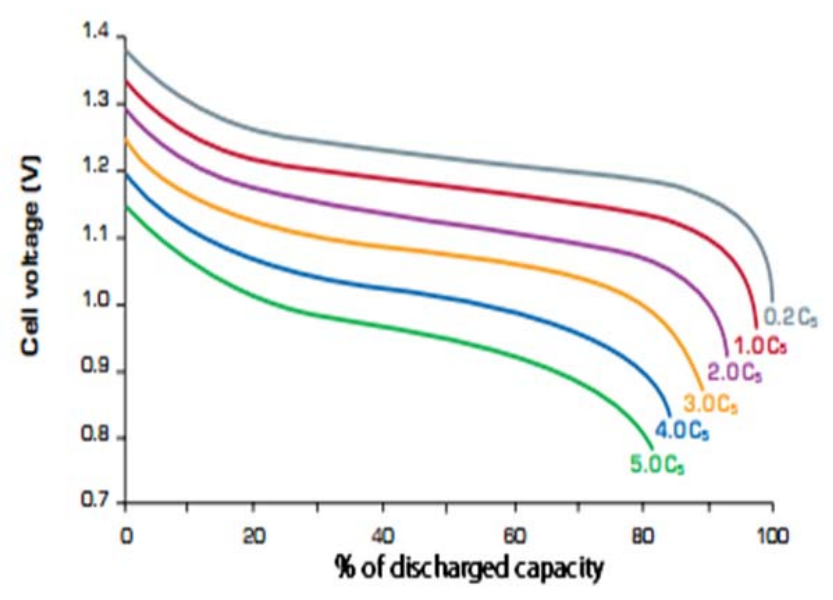

Figure 3. Relationship between the voltage and the capacity at different discharge rate. Source: Saft batteries [10].

$$
\operatorname{SoC}(t)=\operatorname{SoC}(0)-\frac{1}{A h_{\text {nom }}} \int_{0}^{t} I_{m}(t) d t
$$

where SoC $(0)$ is the starting value of SoC and $I_{\mathrm{m}}(t)$ is the measured current.

Charge accumulation techniques where SoC is determined by monitoring battery charge and discharge current are impractical in the long term due to accumulation of errors. For this reason this technique makes it impractical to be used by itself. A monitoring technique combining the opencircuit voltage under no-load condition, and coulometric measurements under constant load has been implemented in [13] on a microcomputerbased circuit. On the other hand, correction factors are required for different discharge rates and ambient temperatures [12].

\subsection{Gas Gauge IC for power-assist applications}

Current based SoC estimation, known as coulomb counting [5], which is currently selected, calculates the state of charge by measuring the instantaneous current of the battery and integrating in time both in the process of charging and discharging. The reason for using this method is because it obtains a good correlation of the measurement data and also because it can be implemented quickly because of the already existing specific integrated circuit (IC). This allows us to rapidly develop a prototype that can be used to estimate the remaining charge left in the battery in Guanay II and to perform some field tests. In this implementation the bq2013 IC (a Gas Gauge IC for PowerAssist Applications) from Texas Instruments Company, specifically designed for $\mathrm{Ni}$-Cd batteries [9] has been used.

Figure 4 shows a block diagram of the SoC estimator where the batteries, motors (load) and charger can be observed. The bq2013 constantly monitors the current flowing through the batteries and also measures the voltage and the internal temperature to compensate for various factors. Finally, the communication to the SoC estimator is through a serial communication, allowing the user to read and write some parameters of the configuration and read the value of the charge of the batteries with the microcontroller.

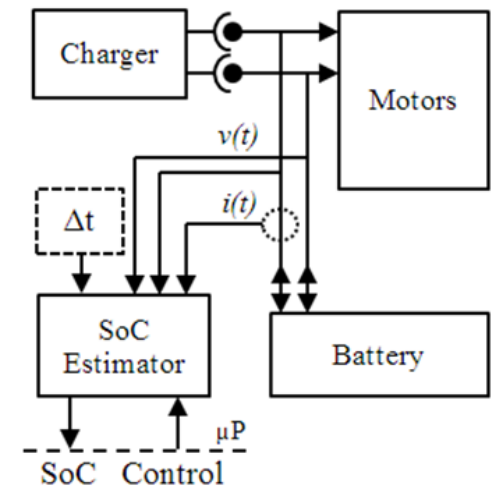

Figure 4. Block diagram of the SoC Estimator. 

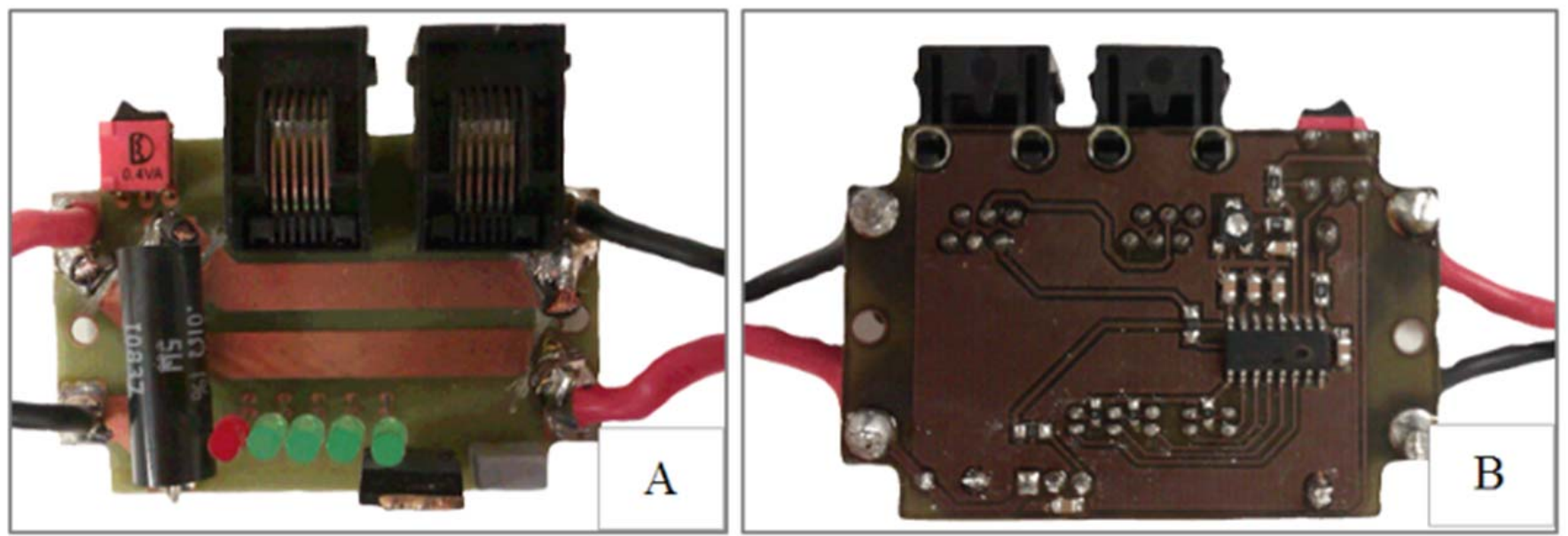

Figure 5 . State of charge measuring prototype. A) Top view B) Bottom view.

\subsection{Prototype}

The prototype developed is shown in figure 5, where the communication ports with the PC-104 and LEDs for visual indication can be seen. This IC includes a simple single-pin serial data interface with command-based protocol (HDQ plus return). For this reason a RS-232 to HDQ interface has been developed to communicate with both gas gauge and PC-104 systems [9].

\subsection{The SoC measurement method}

As explained in section 3.1 there are some drawbacks in using a simple coulomb counting SoC. These drawbacks include the noise, temperature effects, discharge rates and how to estimate the initial SoC value. The algorithm used should include these effects to get a reliable reading of the SoC. The bq2013 implements compensations to minimize these drawbacks. The operational overview diagram in figure 6 illustrates the operation algorithm of the IC.

As can be seen in figure 6, the IC compensates charge current for charge rate and temperature. Discharge current is load compensated and allows automatically adjustment

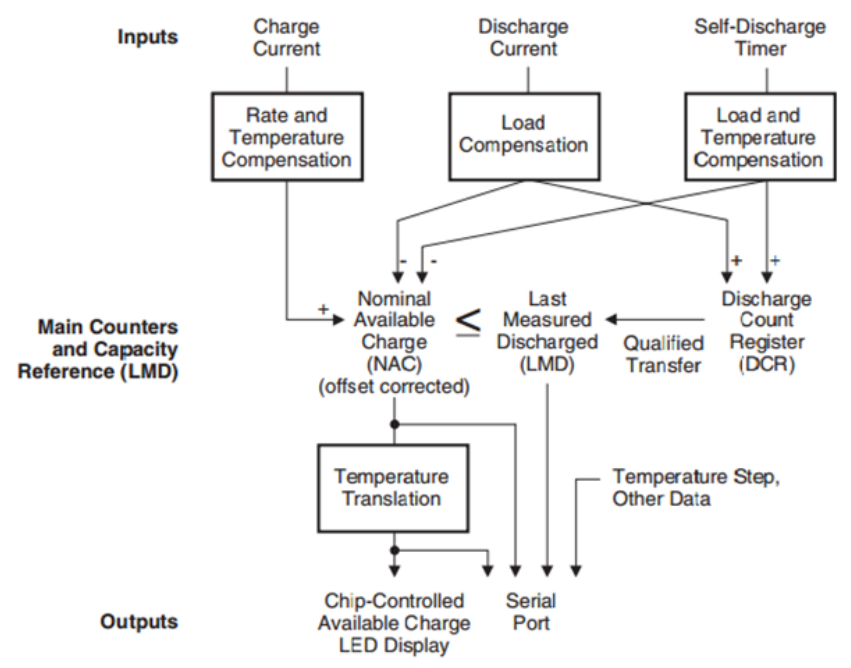

for self-discharge. The main counter, nominal available capacity (NAC), represents the available battery capacity at any given time. Battery charging increments the NAC register, while battery discharging and self-discharge decrease the NAC register and increment the DCR (discharge count register).

The DCR and LMD (last measured discharge) registers are used to update and adjust the initial SoC value.

Texas Instruments provides equation (3) to obtain the value of the internal register NAC (in $\mathrm{mVh}$ ) of the bq2013 IC, which monitors the voltage drop across a resistor connected in series between the battery and ground.

$$
N A C(m V h)=\text { BatteryCap acity }(m A h) \times
$$

$$
\times \text { Senseresis tor }(\Omega) \times \text { Scale }
$$

where Senseresistor $=0.01 \Omega$ and Scale $=640$ (predefined). We can see the relation between the NAC register and the SoC in mAh.

As seen in (3) the NAC value depends on the capacity of the battery and is determined by charging and discharging. Therefore, the consumption can be calculated from expressions (4) and (3):

Consumptio $n(A)=\frac{\Delta \text { BatteryCap acity }(A h)}{\Delta t(h)}$.

Finally, by correcting scale factors, we can obtain the instant consumption using equation (5):

Consumptio $n(A)=\frac{\Delta N A C(m V h)}{\Delta t(s)} \times \frac{3600}{640 \times 1000 \times 0.01}$,

where 3600 is the time factor correction; 640 is scale factor determined by the IC; 1000 is the ampere scale factor correction; and 0.01 is the sense resistor in $\Omega$. 


\section{CHARGING SYSTEM AND BATTERY CONNECTION}

In this section the implementation of charging system and battery connection are described. These two systems can improve the operability of the vehicle and increase safety and reliability. Figure 7 shows a schematic model of this system.

It shows the three battery packs connected in parallel. The switch (SW) between batteries and the AUV electronics is radio-controlled and allows the user to turn on and off the vehicle. Finally, an external charger can be connected using a two pin connector to charge the batteries.

\subsection{Charging system}

The energy of the Guanay II comes from the $2 \mathrm{~s} 3 \mathrm{p}$ battery packs that can provide $24 \mathrm{Vdc}$ and $21 \mathrm{Ah}$. However, a problem arises with this configuration when the batteries have to be charged. Many manufacturers do not recommend charging two battery packs in parallel because this can cause damage especially at high charge rates. Until now, types of chargers that can charge a single pack only were used, but the vehicle must be opened every time. In order to maximize the mission time a system was designed for simultaneous parallel charging of all battery packs through a single external connector. The location of this single point for charging means the it is not necessary to disassemble the vehicle's mechanical and electronic systems and so the charging operation is faster. Figure 8 shows the connector used for charging the batteries. This connector is located in one of the covers of the sealed cylinder. It is manufactured by Subconn Company. Specifically it is a circular 4-pin capable of supporting up to $600 \mathrm{~V}$ and $10 \mathrm{~A}$ at pressures up to 1400 bars.

After discussions with the manufacturer, it has been decided to use a low rate constant voltage charge applied to all the $2 \mathrm{~s} 3 \mathrm{p}$ batteries to prevent damage. A constant charge around $0.2 \mathrm{C}$ is used. By keeping the charge current low enough so that the battery does not generate any heat, this method performs charging without using any control. The calculation formula for semi-constant-voltage charge system is as follows (6):

$V_{C H}=V_{C} \times N \times(k)$,

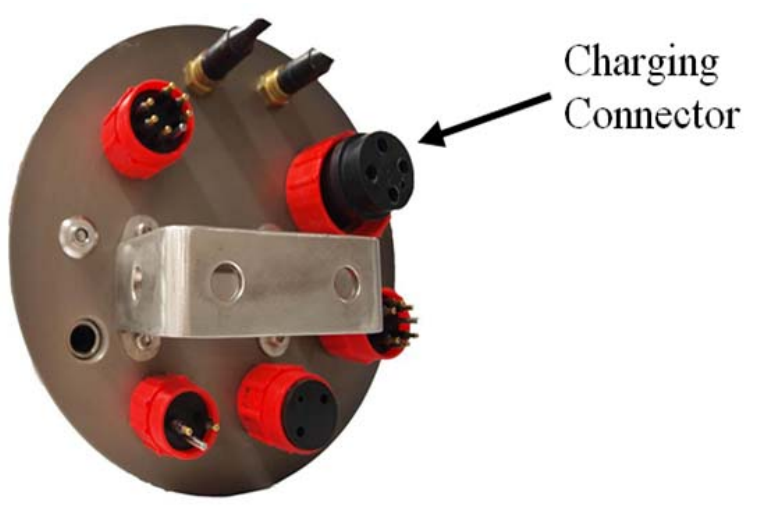

Figure 8. Subconn connector for charging in one of the covers of the sealed cylinder of Guanay II AUV. where: $V_{C H}$ is the output voltage of the DC power supply for charge; $V_{c}$ is the single-cell battery voltage $(1.45 \mathrm{~V} /$ cell: average battery voltage during charge at $\left.20^{\circ} \mathrm{C}, 0.1 \mathrm{C}\right) ; N$ is the number of cells used; $(k)$ is the stabilizing constant and must be selected in accordance with the purpose of the device in which the battery pack is used.

The value of the above-mentioned stabilizing constant (k) must be selected carefully and after discussions with the manufacturer, using a value of 1.25 has been decided upon. Using equation (3) a charging voltage of $29 \mathrm{~V}$ has been obtained. Moreover, the current has been limited to $5 \mathrm{~A}$ to prevent any overcurrent.

Charge rate is often denoted as $\mathrm{C}$ or C-rate and signifies a charge or discharge rate equal to the capacity of a battery in one hour. Equation (7) shows the relation between the capacity of battery in Ah and the current in A.

$$
\operatorname{CapacityofBattery}(A h) \times C(1 / h)=\operatorname{Current}(A)
$$

\subsection{Battery connection}

In order to have control and easy access to the battery connection/disconnection to electronics and propulsion of the vehicle, a wireless device that acts as a switch has been incorporated. Figure 7 shows a schematic model of this system.

The user can turn off/on the switch of the vehicle using the remote controller. This operation enables the connection or disconnection of the batteries while the AUV is in the water, obtaining greater security in the vehicle. A HIR6-433 RF AM $433 \mathrm{MHz}$ receiver/decoder from Rfsolutions has been used for this purpose. These types of modules provide a very low power receiver, combined with a flash programmable controller, supplied pre-programmed to operate with the Keeloq Transmitter encoder. The range from such a system can be up to 50 meters Line of Sight (LOS).

Finally, a new antenna with epoxy resin has been designed to allocate the HIR6-433 and provides the necessary protection in the water and in the depth. Besides hosting this module, the antenna is also used to install a new WiFi communication module and GPS antenna.

\section{EXPERIMENTAL RESULTS}

Initially both systems, the connection/disconnection of the battery and its charging and SoC estimation have been tested in the laboratory, where the proper functioning of all the systems designed was verified.

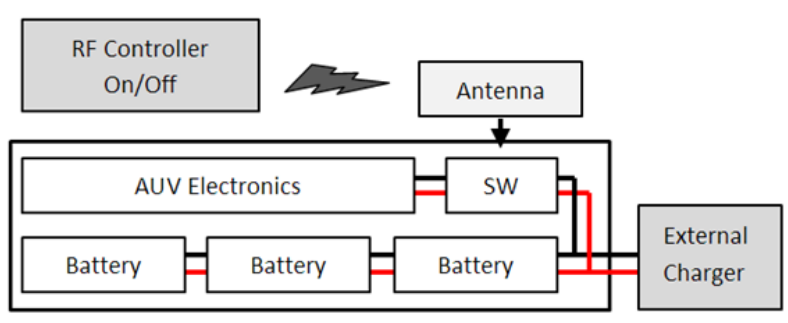

Figure 7. Scheme of charging system and battery connection. 


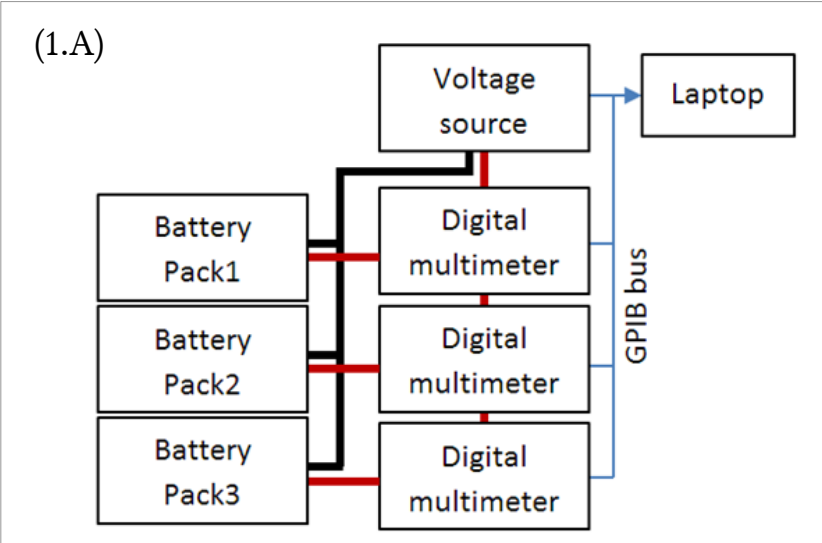

(2.A)

$(1 . \mathrm{B})$
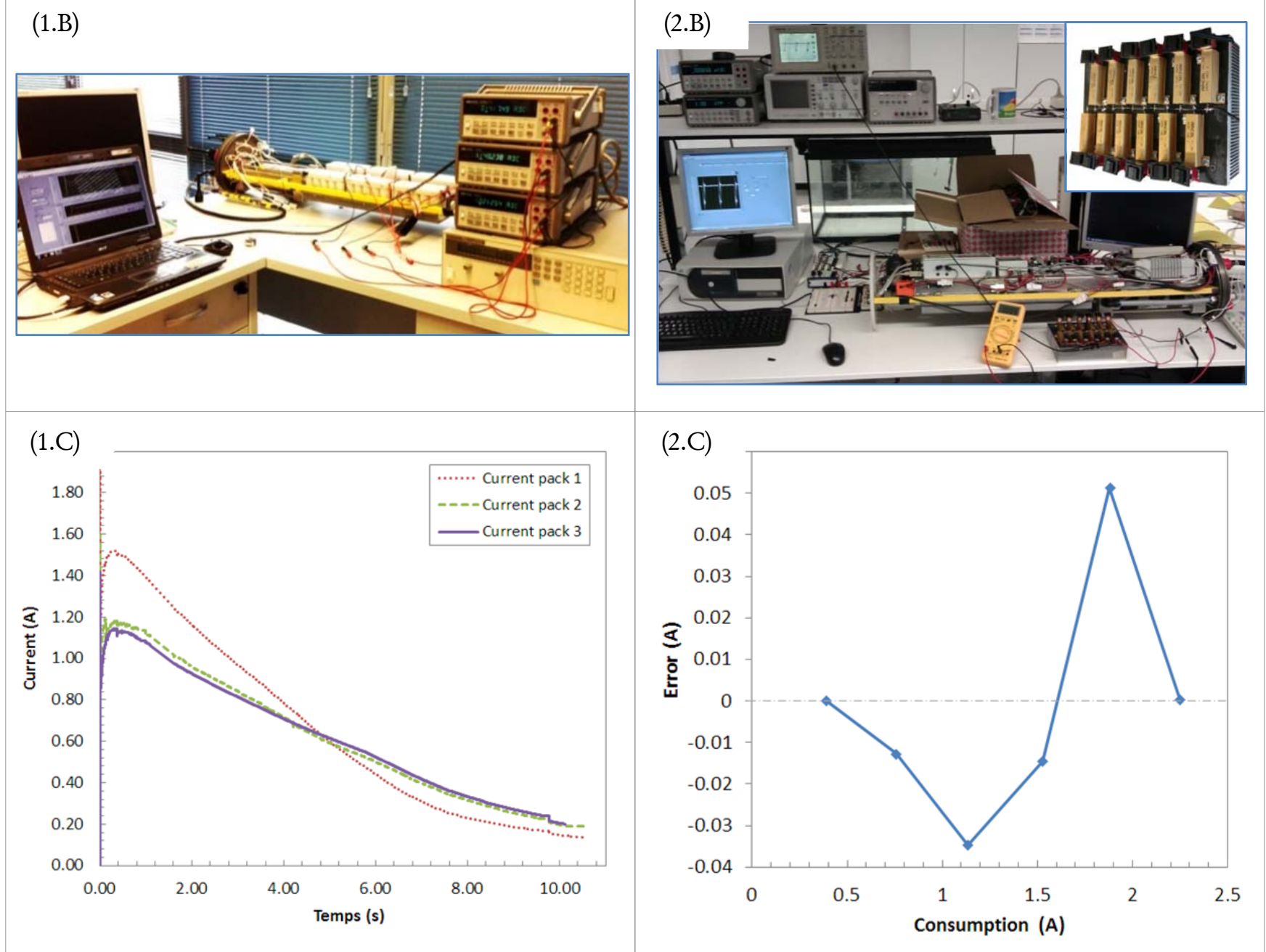

Figure 9. shows the two laboratory tests. In $1 \mathrm{a}$ charge testing of all packs of batteries in parallel can be seen. In $2 \mathrm{~b}$ discharge testing and a comparison between sensed current from the multimeter and sensed current from the bq2013 can be observed.

1.A) Block scheme of the battery charge test. Three battery packs, three digital multimeters, one voltage source and one laptop to monitor the variables. All of them are connected through a GPIB port.

1.B) Shows the test bench where the battery charging has been tested.

1.C) The current of the three packs (red, green and purple) of batteries during a low rate charging in parallel.

2.A) Block scheme of the battery discharge test. Five resistors of 18 ohms in parallel as a load have been used. The three measurements (from the ammeter, from the bq2013 and theoretical) have been compared using a laptop.

2.B) Test bench that has been used to check and adjust the SoC sensor of the battery. For this laboratory experiment a bank of five loads in parallel has been used. Each load of 18 ohms introduces a $433 \mathrm{~mA}$ of consumption current.

2.C) Vehicle current measurement error using the SoC circuit (X-axis is the total current of the vehicle). 


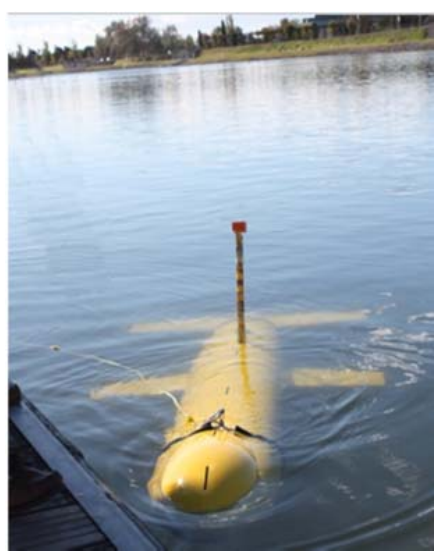

Figure 10. Guanay II AUV in the field test.

One of the most critical parts is the parallel charging of battery packs, in particular the individual currents of each pack. Laboratory tests have been conducted to validate the correct performance of the charging. In this test a power supply and three digital multimeters to monitor individual currents of each pack during charging has been used (see figure 9 point 1.A, Block scheme). Figure 9 in point 1.B shows the test bench where the battery charge has been tested. For this purpose, three ammeters and one power supply connected to a computer by GPIB have been used. This computer is responsible for controlling and saving instrument data.

Figure 9 in point 1.C shows the current of the three packs of batteries during charging in parallel mode. In this picture it can be seen that after 10 hours of charge the current drops to $0.15 \mathrm{~A}$ and the battery is practically charged. Also, the difference of the current charge in one of the packs can be observed. This is one reason why the high rate current charge is not recommended in order to prevent damages. In figure 9 point 1.C the rate of current used to charge the batteries can also be seen. According to the equation (7) a maximum current rate of $0.17 \mathrm{C}$ is obtained during the first hour of charge, from which this rate decreases to less than $0.05 \mathrm{C}$.

On the other hand, as seen in equation (5), the current consumption of the vehicle can be calculated using the $\mathrm{SoC}$

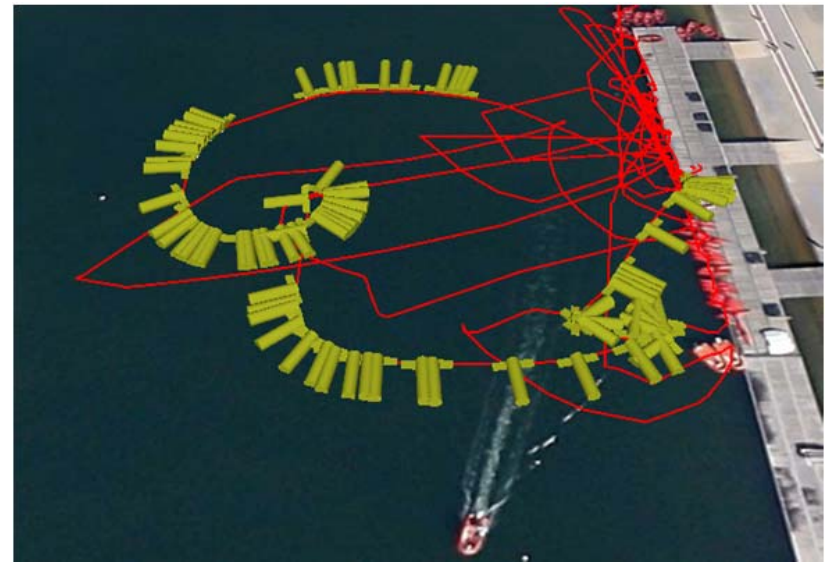

Figure 11. Different trajectories performed by the vehicle during the field test.

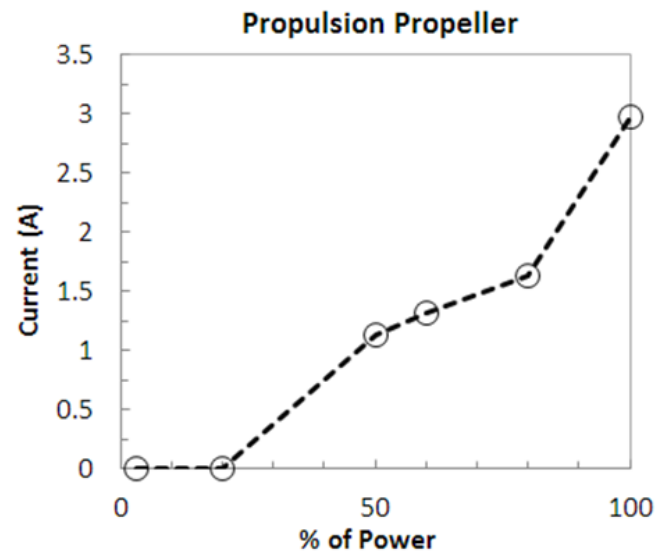

Figure 12. Percentage of power to the engine requested vs. current drawn. (Propulsion propeller).

circuit. A test and a calibration have been conducted to validate the system, using a digital multimeter and five 18 ohms resistors as a load (see figure 9 point 2.A, Block scheme). Figure 9 in point 2.B shows the test bench that has been used to check and adjust the SoC sensor of the battery. For this laboratory experiment a bank of five loads in parallel has been used. Each load of $18 \mathrm{ohms}$ introduces $433 \mathrm{~mA}$ of consumption current.

Figure 9 point 2.C shows the vehicle's current measurement error using the SoC circuit bq2013. The error varies according to the total current of the vehicle with less than $\pm 50 \mathrm{~mA}$. Therefore, using the NAC register a sufficiently accurate method to estimate the power consumption from Guanay II has been obtained.

Afterwards, these systems have been validated in various vehicle navigation tests. For example, one of these tests was performed at the Canal Olimpic de Catalunya in Castelldefels (The Olympic channel located in Castelldefels) as shown in figure 10. Figure 11 shows different trajectories performed by the vehicle during the field test.

Figure 16 shows the instantaneous battery consumption due to the action of the propulsion engine and two direction motors of the vehicle during a path on the water. The first three graphs show the action of propellers in \%

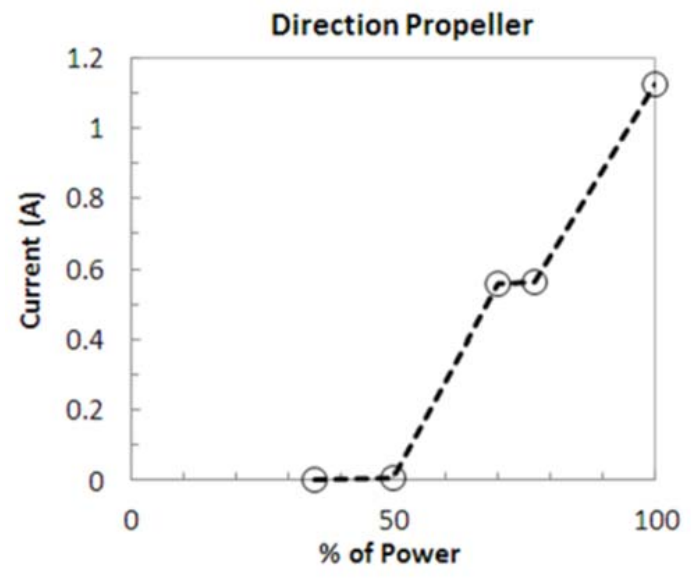

Figure 13. Percentage of power to the engine requested vs. current drawn. (Direction propeller). 

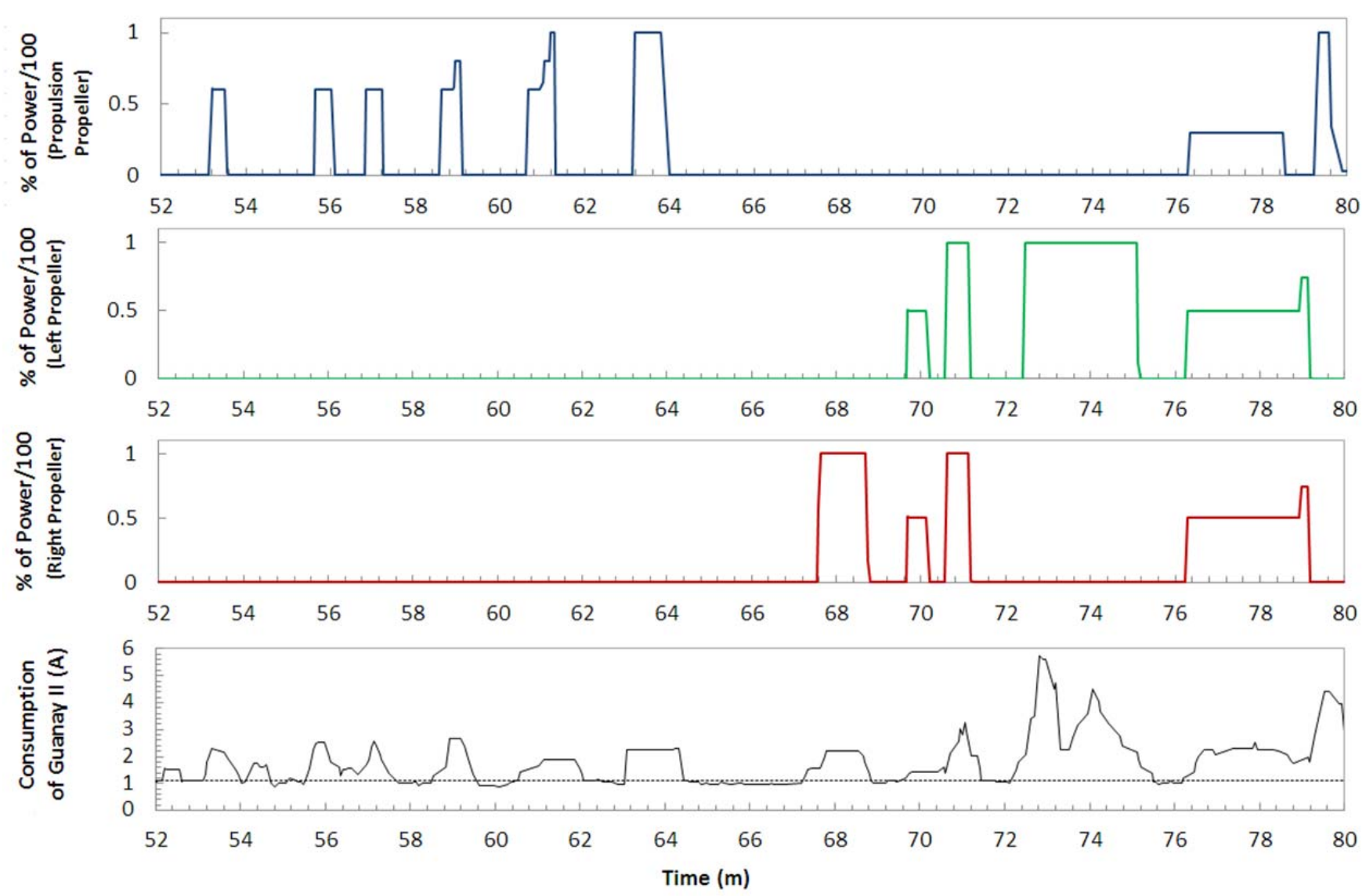

Figure 14. Consumption of the vehicle versus power of the propellers.

(up to one). In blue the action of the main propeller is shown and in red and green the action of side propellers is shown. The fourth graph shows the instantaneous battery consumption (black line) and the mean of this (dashed line).

One can observe the relationship between consumption and the action of the engines. Also, the average consumption from the remaining electronic elements (PC104, radio modem, GPS, etc.) can be seen. This value may be contrasted with the consumption values of table 1 . The difference may come due to the time of use of the radio modem or PC104. In any case, this can be a field to investigate in future work.

From the experimental results shown in figure 14, the relationship between the percentage of power to the engine requested and the current drawn has been made. Figure 13 shows this relationship for the left direction propeller and figure 12 for the propulsion propeller.

The results show that, at low power, the engine will not draw power and therefore will not work. This is because a minimum amount of power is needed to run the motors. For the direction propellers more than 50\% of power has been required and for the propulsion propeller more than $20 \%$. However, more field test points are needed to obtain a more accurate relationship.

These tests also confirmed that disconnecting the batteries when the vehicle is still in the water provides security at its landing ground.

\section{CONCLUSIONS}

A system for measurement and for energy management for an underwater vehicle have been designed. The system monitors the state of charge of the batteries and the instantaneous consumption of the thrusters, information that the control mission needs to optimize and facilitate navigation. A single external access connector in the vehicle allows all batteries to be charged and a radio frequency control switch is used to connect and disconnect the vehicle's power supply even in water. The navigation tests performed confirm that the system operates correctly and the data measured is reliable.

\section{ACKNOWLEDGEMENT}

This work was supported by the Spanish Ministry of Economy and Competitiveness under the research project: "Sistemas Inalambricos para la Extension de Observatorios Submarinos" (CTM2010-15459).

\section{REFERENCES}

[1] J. Timothy Pennington and F. P. Chavez, "Seasonal fluctuations of temperature, salinity, nitrate, chlorophyll and primary production at station h3/m1 over 1989-1996 in Monterey bay, California” Deep Sea Research Part II: 
Topical Studies in Oceanography, vol. 47, no.5, pp. 947-973, 2000.

[2] S. Gomáriz, J. González, A. Arbos, I. Masmitja, G. Masmitja, J. Prat. "Design and construction of the Guanay-II Autonomous Underwater Vehicle". Oceans 2011 IEEE/OES. Santander, Spain. June 2011.

[3] S. Gomáriz, J. Prat, A. Arbos, O. Pallares, and C. Viñolo, "Autonomous vehicle development for vertical submarine observation" in International Workshop on Marine Technology, (Vilanova i la Geltrú, Spain), november 2009.

[4] S. Gomáriz, J. Prat, P. Gayà, and J. del Río, "Development of low-cost autonomous oceanographic observation vehicle", in Oceans'09 MTS/IEEE, (Brement, Germany), may 2009.

[5] F.Codecà, S. M. Savaresi, V. Manzoni. "The mix estimation algorithm for battery State-of-Charge estimator - Analysis of the sensitivity to measurement errors" in Join 48th IEEE Conference on Decision and Control and 28th Chinese Control Conference Shangahai, P.R. China, December 1618, 2009.

[6] M. Gonzlaez, M.A. Perrez, J.C. Viera, C. Carballo, A. Garrido. "A new, reliable and easily implemented $\mathrm{NiCd} / \mathrm{NiMH}$ battery state estimation method". Instrumentation and Measurement Technology Conference, 1999. IMTC/99. Proceedings of the $16^{\text {th }}$ IEEE. Page(s): 1260 -1264 vol.2.
[7] N. Kato, K. Yamamoto. "Estimation of the capacity of nickel-cadmium batteries by measuring impedance using electrolyte-deficient battery characteristics" Telecommunications Energy Conference, 1995. INTELEC '95. 10.1109/INTLEC.1995.499046 .

[8] I. Damlund. "Analysis and interpretation of ACmeasurement on batteries used to assess state-of-health and capacity-condition" Telecommunications Energy Conference, $1995 . \quad$ INTELEC '95. 10.1109/INTLEC.1995.499055.

[9] Texas Instruments. "HDQ Communication Basics". Application Report. SLUA408A - December 2006 - Revised September 2008.

[10] Saft Batteries.

http://www.saftbatteries.com/Technologies_Nickel_NiCd_ 293/Language/en-US/Default.aspx. May 2013

[11] Y. Çadirci, Y. Özkazanç. "Microcontroller-based on-line state-of-charge estimator for sealed lead-acid batteries" Journal of Power Sources 129 (2004) 330-342

[12] Chyuan-Yow Tseng, Chiu-Feng Lin. "Estimation of the state-of-charge of lead-acid batteries used in electric scooters" Journal of Power Sources 147 (2005) 282-287.

[13] J.H. Aylor, A. Thieme, B.W. Johnson. "A battery state-ofcharge indicator for electric wheelchairs”. IEEE Trans. Ind. Electron. 39 (5) (1992) 398-409. 